\title{
Evaluación del control interno del área de Inventarios en la fábrica de puros ACCSA
}

\author{
Florencio Germán Pérez Úbeda ${ }^{1}$
}

1 Egresado de la maestría en contabilidad con énfasis en auditoria UNAN-Managua FAREM-Estelí.

Correo electrónico: germanbanban@yahoo.es

\section{RESUMEN}

El presente trabajo estuvo encauzado a la Evaluación del control interno del área de inventario de materia prima y productos terminados en la fábrica de puros ACCSA, mediante el análisis de los cinco componentes del informe COSO. Este estudio se realizó tomando en consideración el enfoque cuali-cuantitativo de tipo descriptivo y de corte transversal. La muestra utilizada fue de siete funcionarios de la empresa que están directamente relacionados con el área de inventarios. La información se obtuvo utilizando técnicas de levantamiento de datos, tales como: entrevistas, encuestas y revisión documental. Estos dieron un mejor entendimiento sobre los procedimientos que se llevan a cabo en la fábrica respecto a la administración y manejo del inventario de materiales y mercancías.

Los resultados obtenidos indican que los controles internos de inventarios que se aplican en la fábrica son ineficientes, ocasionando problemas de segregación de funciones que derivan en incertidumbre en cuanto a la realización de las operaciones de inventario de manera efectiva y poniendo en duda la confiabilidad de la información financiera.

Palabras clave: Control interno, inventarios, segregación de funciones.

\section{ABSTRACT}

This work been addressed to the Evaluation of internal controls carried out in the area of materials and goods inventory of the cigar manufacturing company ACCSA, through the analysis of the five components of COSO report. For this purpose the study was developed taking into account the qualitative and quantitative approach, descriptive and cross-sectional. The sample used was obtained from a staff of seven employees that are directly related to the inventory area. Information was gathered using data collection techniques, such as interviews, inquiries and documentary research. This provided a better understanding of the procedures carried out in the factory for the administration and management of the inventory of materials and goods, as well as the employee's commitment and their duties related to these procedures.

The survey results indicate that inventory internal controls carried out at the factory turn to be ineffective and cause problems of segregation of duties generating doubts on the proper execution of inventory operations and questioning the reliability of the financial information coming from the area.

Keywords: Internal control, inventories, segregation of duties. 


\section{INTRODUCCIÓN}

En los últimos años las organizaciones le han dado un alto valor al hecho de poseer un buen sistema de control interno. Esto debido a lo fácil y práctico que resulta medir la eficiencia y productividad al momento de implantarlos, sobre todo si estos se centran en las actividades básicas que ellos realizan.

El inventario en la fábrica de Puros American Caribbean es uno de los activos más importantes y uno de sus elementos más complejos y de mayor valor. Se constituye en un elemento fundamental para el cálculo de los costos de fabricación y ventas y por consiguiente de los resultados de la empresa. Al ser el inventario de un producto manufacturado, su complejidad es mayor y el manejo de los mismos requiere un análisis y una evaluación minuciosa. Por las características de estos inventarios podemos afirmar que es uno de los activos que está más expuesto a manipulación, lo que puede conllevar a deterioro, daños por manejo inadecuado, robos etc. Igualmente los controles físicos y el registro de los mismos representan un factor importante a tomar en cuenta para la preparación de la información financiera y la toma de decisiones.

En este particular los controles se vuelven una herramienta fundamental, porque aunque la empresa cuente con buenos planes, una buena estructura organizacional y una dirección eficiente, esta no podrá determinar cuál es la situación real de la organización si no se cuenta con un mecanismo que verifique e informe si lo real va de acuerdo con los objetivos. En ese sentido este estudio está dirigido a evaluar los controles internos del área de inventarios en la fábrica American Caribbean Cigars.

\section{MATERIALES Y MÉTODOS}

El presente estudio "Evaluación del control interno en el área de Inventario en la Fábrica de Puros American Caribbean S.A en el año 2011" se realizó mediante una investigación de naturaleza descriptiva, pues consistió en conocer las situaciones, costumbres y actitudes predominantes a través de la descripción de las actividades, objetos, procedimientos y personas involucradas directa e indirectamente en el área de inventarios.

El enfoque es cuali-cuantitativo debido a que se analizó un número reducido de objetos de investigación y a la vez se utilizó un programa informático para su formalización.

Con la investigación se identificó las características de los procedimientos administrativos, políticas y normas que rigen las tareas de los empleados que trabajan en el área de inventario.

La información fue recolectada de manera directa mediante encuestas y entrevistas al personal clave involucrado directamente e indirectamente en el área de inventariaros de la fábrica, así como la revisión documental.

Asimismo se obtuvo información a través de entrevistas a dos expertos en el rubro del tabaco y mediante investigaciones realizadas en el área de inventarios y textos referidos al tema.

\section{RESULTADOS}

Con este estudio se logró identificar que las actividades para el manejo de los inventarios se llevadas a cabo y amparadas en la experiencia de los involucrados. Básicamente el trabajo se realiza sin una guía que facilite, oriente, establezca límites de actuación, normas y políticas sobre cómo se debe desempeñar esta tarea.

El ambiente de control de la empresa presenta deficiencias en la forma en cómo se estructuran las actividades del negocio, entre ellas: no cuentan con un manual de funciones escrito por lo que el 
nivel de competencia del personal no está bien establecido. Carecen de organigrama estructural de la empresa, lo que dificulta la organización, el control de las operaciones y que se conozcan las líneas de autoridad.

A pesar de que los directivos transmiten y enfatizan en la importancia de tener un comportamiento ético y de integridad, la fábrica carece de un código de comportamiento y ética que refuerce y fortalezca esta actividad.

En relación a la evaluación de los riesgos la mayor debilidad se encuentra en la falta de análisis de estos, ya que los directivos no evalúan las probabilidades de ocurrencia de los riesgos, ni tienen estimados los efectos de los mismos. Lo que significa que los controles utilizados son más correctivos que preventivos.

Un punto de suma importancia es el hecho de que la fábrica no posee una póliza de seguros para la protección de los inventarios, lo que significa que ellos no están estimando correctamente la posibilidad de un evento fortuito que los pueda afectar.

Por otro lado nunca se les ha practicado ningún tipo de auditoría externa que les ayude a fortalecer y mejorar los controles internos.

En cuanto a las actividades de control American Caribbean registra sus operaciones de inventario en tiempo y forma y presenta sus estados financieros de manera oportuna. Sin embargo es importante señalar que es necesario segregar correctamente las funciones de autorización, registro y custodia para asegurar la validez de la información.

No poseen un manual de control interno de inventarios por lo que los empleados trabajan sin una guía que los oriente a través de políticas de manejo de inventarios y les establezca límites de actuación.
En referencia al componente de información y comunicación el aspecto fundamental reside en el sistema de información utilizado actualmente por la fábrica. Hoy por hoy el sistema de información utilizado cumple con las expectativas de la fábrica sin embargo con los cambios propuestos en la estructura organizacional se verán en la obligación de realizar cambios en el sistema.

En lo que concierne al componente de monitoreo y supervisión lo más destacado es la falta de análisis periódico del funcionamiento de los controles internos de los inventarios y la inexistencia de controles gerenciales que les permita conocer el nivel de eficacia los mismos. La gerencia de la fábrica juega un rol protagónico en la eficiencia o deficiencia de los controles internos ya que existe una relación directa entre las actividades de monitoreo y supervisión y la eficacia de los controles internos. En este sentido podemos señalar a la gerencia como responsable directo de las deficiencias encontradas.

\section{RECOMENDACIONES}

En relación a los resultados del trabajo investigativo sobre la evaluación del control interno de inventarios de la fábrica "American Caribbean Cigars. S.A", se dieron las siguientes recomendaciones para que la gerencia evaluara la posibilidad de implementarlas:

- La gerencia en conjunto con administración y recursos humanos debe elaborar un código de ética que fije normas que regulen el comportamiento de los empleados y establezca sanciones disciplinarias por el no cumplimiento de las mismas.

- La gerencia en conjunto, con administración y el responsable de recursos humanos deben diseñar y estructurar el organigrama de la fábrica 
que precise las áreas funcionales, las líneas de autoridad, y defina las relaciones de subordinación y comunicación.

- La gerencia en conjunto, con administración y el responsable de recursos humanos deben establecer la misión, visión y valores de la fábrica que den a conocer a los empleados actuales y las nuevas contrataciones, la labor de la fábrica, las metas a futuro y el comportamiento requerido.

- La gerencia en conjunto con administración y recursos humanos debe elaborar el manual de funciones que establezca responsabilidades de cada área de la fábrica y establezca todos y cada uno de los procesos de las mismas. Debe contener la Visión, Misión y valores de la fábrica.

- La gerencia debe contemplar la contratación de un seguro para la protección de los inventarios en bodega.

- La gerencia debe contemplar la contratación de un contador y un responsable de bodega. Las funciones de autorización, custodia y registro en las actividades de manejo de inventarios y compra de materiales deben definirse correctamente para evitar que un solo empleado realice un mismo proceso en todas sus fases.

- La gerencia y administración deben contemplar la construcción de una bodega de productos terminados, para garantizar la salvaguarda de los puros.

- La gerencia y administración deben elaborar un manual de control interno de inventarios que contenga los lineamientos, procedimientos y políticas a seguir en el área de inventarios de materiales y mercancías.

\section{BIBLIOGRAFÍA}

Anaya Tejero, Julio Juan (2008). Almacenes, análisis, diseño y organización. ESIC Editorial, España.

Arens, Alvin y Loebbecke, James (1996). Auditoría un enfoque integral. Sexta edición. Prentice Hall Hispanoamericana, S.A México D.F.

Coopers y Lybrand (1997). Los nuevos conceptos del control interno (Informe Coso), España, Ediciones Díaz de Santos S.A.

Cuevas Villegas, Carlos (2001). Contabilidad de Costos, enfoque gerencial y de gestión. Pearson Educación de Colombia.

Estupiñan Gaitán, Rodrigo (2006). Control Interno y fraudes, segunda edición, ECOE EDICIONES. Bogotá, Colombia.

Fonseca Luna, Oswaldo (2011). Sistemas de control interno para organizaciones. Primera Edición. Imprenta Publicidad \&Matiz Lima Perú.

Fonseca Luna, Oswaldo (2008). Vademécum Contralor. Primera edición, Imprenta Publicidad \& Matiz. Perú.

González, Araceli; Cabrales, Damaris (2010). Evaluación del sistema de control Interno. Las Tunas. Cuba

González Dalmau; Lorgio, Ángel (2012). El Derecho en la Aplicación de la contabilidad forense. Editorial Académica Española.

Hernández y Rodríguez, Sergio (2006). Introducción a la Administración. Editorial McGrawHill. Cuarta Edición. México DF.

Hitt, Michael; Black Stewart, Porter Lyman (2005). Administración. Prentice Hall.

Horngren, Charles (2002). Contabilidad. Editorial Prentice Hall. Quinta edición. México.

Koonts, Harold (1998). Administración una perspectiva global. Editorial McGraw Hill. Onceava edición, México D.F.

Mantilla B, Samuel Alberto. Control Interno Informe Coso, Cuarta Edición, Colombia, Editorial ECOE EDICIONES 2005. 
Mantilla B, Samuel Alberto (1998). Control Interno de los nuevos instrumentos financieros, ECOE EDICIONES, Colombia.

Muller, Max (2004). Fundamentos de Administración de inventarios, Editorial Norma.

Perdomo Moreno, Abraham (2000). Fundamentos de control Interno. Editorial Thomson. Novena Edición.

Peña Bermúdez, Jesús María (2000). Control, Auditoria y Revisoría Fiscal Tercera Edición, ECO Ediciones.

Peterson Vejar, Gastón (2002). Contabilidad de costos por procesos. Universidad Autónoma de Baja California.

Polimeni, Ralph (1994). Contabilidad de Costos. Editorial McGraw Hill. Tercera Edición. Santa Fe de Bogotá, Colombia. 